ON

\title{
MALIGNANT TUMOURS,
}

CONNECTED WITH THE HEART AND LUNGS.

BY JOHN SIMS, M.D.

PHYSICIAN TO THE ST. MARY-LE-BONE INFIRMARY.

READ JANUARY 22ND, 1833.

To illustrate the origin and growth of tumours, or adventitious formations in the various organs of the body, is at present an object exciting the diligent investigation of pathologists. Several valuable papers on tumours of a malignant character, have been published in the preceding volumes of the Transactions, and in other works, comprising instances of the disease in many of the tissues and organs: but comparatively few cases are recorded, where the heart was involved in the morbid growth.

The first two cases which I shall relate, belong to the class of Malignant Diseases, whose varieties have obtained the names of fungus hæmatodes, medullary sarcoma, soft cancer, \&c. They present circumstances, in their history and progress, which will probably be interesting to the Society. 


\section{A SE I.}

Malignant tumour attached to the heart and lungs, and to the uterus and its appendages, with phlegmasia dolens of the right upper extremity.

M. K. residing in Musenm Street, a tall, wellformed young person, aged 23, had enjoyed tolerably good health, till near the period when her present disease was first noticed. She has recently suffered great difficulty of breathing, frequent cough, and considerable pain in the chest, with other symptoms, supposed to indicate a severe inflammatory affection of the lungs. The usual remedies were had recourse to for her relief, but the symptoms did not give way to the treatment adopted, and in a short time, others appeared of a more formidable character.

In addition to the foregoing symptoms, she mentioned, at the time of my first visiting her, the presence of some-swelling, in the lower part of the abdomen, and on examination, several distinct and large tumours could be felt rising out of the pelvis, and probably connected with the sexual organs. Above the clavicle, and along the blood-vessel of the right side of the neck, there was a number of enlarged lymphatic glands of various sizes.

Her disease made a rapid progress, and in a short time, fluid began to collect in the cavity of the peri- 
toneum, the tumours rose higher, and increased in magnitude; the lower extremities became edematous. The distress in breathing increased, and also the cough, but no expectoration of pus, or other signs of ordinary pulmonary tubercles presented themselves. A remarkable symptom now occurred: the sounds of the ventricles were perceived in their usual situation, but the impulse of one or both ventricles was equally distinct over a considerable part of the right side of the thorax anteriorly. Her right arm became painful and enormously swoln, presenting all the signs of phlegmasia dolens from inflamed veins.

From the greatly impeded respiration, the swelling of the abdomen, lower extremities, and right upper extremity, she was unable to vary the position from that of constantly lying on the back : in consequence of this, a large slough took place over the sacrum, which, added to the rapid progress of the disease in the thorax and abdomen, soon terminated her extreme sufferings.

On considering the great difficulty of respiration, the impulse of the heart peculiarly felt over the right side of the thorax, the tumours above the clavicle, and along the blood-vessels of the neck, the phlegmasia dolens of the right upper extremity, the tumours of rapid growth within the abdomen, and at the same time the absence of signs of ordinary disease of the lungs and heart, or, in other words, trying the diseased state of the thorax by seclusion, 
the supposition was justifiable that a large tumour, probably of a malignant or fungoid character, was situated in the right side of the thorax, and attached to the lungs and heart. The result of the dissection proved the correctness of this opinion.

I need not relate the various means that were used to mitigate the sufferings of this young person. I may however remark, that needle punctures, made along the inner side of the upper extremity, materially relieved the pain and nearly reduced the swelling: for, as will be shewn, this was a true specimen of phlegmasia dolens, and I am not aware that this remedy is had recourse to in the analogous affection of puerperal women.

\section{Dissection.}

\section{Head not examined.}

Thorax.-On opening the thorax, a tumour of very considerable size was found imbedded in the right lung, it was closely attached to the great vessels at the base of the heart; it was moveable within the thorax.

On making sections through it, some portions appeared firm and fibrous, and others softer and brainlike. Its colour was of a dirty white, intermixed with streaks of a lead colour, apparently in the direction of its few blood-vessels. It closely involved the 
bronchi and blood-vessels at the root of the right lung, and was firmly attached to the pericardium and vessels immediately issuing from the heart. Circumstances did not allow me to remove the tumour.

Nothing remarkable was observed in the left lung or the texture of the heart.

On dissecting out the right subclavian vein, the preparation of which is now before the Society, it was found to be filled with successive layers of fibrine, the product of inflammation, and the valves at its junction with the jugular are seen distended with this deposit.

The cavity of the peritoneum contained several pints of fluid. The viscera had a deep leaden hue, and there was a remarkably strong exhalation of carburetted hydrogen gas. There were several very large tumours attached to the uterus and its appendages, some of them the size of large oranges : they were soft, and their texture was exactly analogous to the tumour found in the lung.

\section{$C A S E \quad I I$.}

Malignant tumour affecting the right lung, and penetrating the left auricle of the heart.

John Imber, æt. 43, of middle stature, broad chest, and generally athletic appearance, a baker. 
About twelve months ago he applied to me, suffering under an attack of hæmoptysis, attended with impeded respiration, cough, and the symptoms commonly accompanying a loaded state of the blood-vessels of the lungs. The hæmorrhage was occasionally in large quantity, the dyspnœa and oppression were severe, but not such as to prevent his continuing his occupation, except during the occasional severity of his symptoms. I saw him in several of these attacks of hæmoptysis. He was very much relieved by general and local bleeding, digitalis, blisters, \&c. together with a regulated diet.

I lost sight of him for several months until he was admitted into the St. Mary-le-bone Infirmary, Oct. 30, 1832.

His symptoms during this interval had undergone a material alteration for the worse. The difficulty of breathing and sense of oppression in the thorax were much increased: he had had several returns of hæmoptysis : his cough was occasionally very troublesome, with mucous expectoration. A considerable part of the right side of the thorax anteriorly is dull on percussion, and respiration is not heard by the stethoscope. The jugular veins are dilated to three times their usual size, and with part of the subclavian, present large tumours above the clavicles, alternately increasing and diminishing in size: his face is swoln: he complains of severe head ache: pulse sharp and ringing, but neither strong nor very frequent: little loss of flesh: bowels costive. 
After his admission into the hospital, bleeding; blisters, tartar emetic ointment, evidently afforded him relief, and mitigated his sufferings : but this relief was transient. Digitalis and ipecacuanha were given, but the former was soon discontinued, in consequence of its narcotic effects.

Dec. 15. Difficulty of breathing and swelling of the face considerably increased. He was again relieved by small doses of mercury. A few days prior to his death, the dyspnoea was much increased, and all his symptoms were greatly aggravated; he died on the 28th.

A review of the history and progress of this patient's symptoms, the hæmoptysis, extreme difficulty of breathing, and latterly the turgid state of the jugular and subclavian veins, swoln face, head-ache, dullness of the right side of the thorax, the occasional continuance of hæmorrhage, the comparatively little loss of flesh, the absence of the signs of the common diseases of the chest, together with a recollection of the case of M. K., which I have just related, led to the belief that a tumour of considerable size, and of rapid growth, existed in the right cavity of the thorax, which was confirmed by dissection.

Dissection twenty hours after death :-

Head. - The aracnoid was more opaque than usual : there was a considerable quantity of fluid in the sub-aracnoid tissue, and the convolutions were 
consequently separated from each other : about one ounce of fluid in the ventricles. Brain firm and natural.

Thorax.-On raising the anterior parietes of the thorax, a portion of the tumour, several inches in circumference, came into view on the right side. The tumours of the right lung occupied about two thirds of the capacity of the entire thorax. The diaphragm was lower than usual, the space for the left lung was encroached upon by the contents of the opposite side. The heart was situated several inches lower than usual, and pushed much beyond the mesial line.

The contents of the thorax, the liver and diaphragm were now removed from the body.

Left lung.-The left lung was free from adhesion, and the pleura of natural appearance; there was much black matter deposited, and some emphysema. This lung was considerably indurated in some parts, which, on being cut into, consisted of extensive red hepatization. On a careful examination of the lung it was found to be quite free from the morbid growth contained in the other cavity of the thorax: the larger vessels and the bronchi were not engaged in the disease, although the tumours in the left auricle lay close to them.

Right lung.-Pleura much thickened. This lung occupied a considerable space, for the augmented 
contents of the thorax had encroached upon the cavity of the abdomen. A great proportion of it was consolidated, apparently in consequence of old hepatization; in some parts the substance crumbled on the application of gentle pressure, this portion was of a dark or dusky red colour; there was pus in a few small cavities in the section. A small portion was comparatively healthy, and in degree fit for the purposes of respiration. The tumour was extensively attached to this lung, and portions had insinuated themselves between the larger vessels and the carnifications of the bronchi.

The trachea was so pressed upon by the tumour as to render the musculo-membranous part quite flat, and to expand the cartilages into a much wider arch. The bronchi, at the bifurcation, were much dilated; the right bronchus, with several of its subdivisions, passed directly through the tumour.

Most of the bronchial glands are healthy, some of them are shoved forwards, and situated on the surface of the tumour; others near the bifurcation are extended or flattened out over the softer parts of the tumour.

Heart.-The pericardium was much dilated at the base from several large tumours being developed within it.

Right auricle.-The tumour presses upon the voL. XVIII. 
posterior part of the auricle so as to burst it inwards, and in one part a small tubercle has penetrated, the size of a split bean. The cavity of the auricle is much dilated.

The inferior vena cava was not connected with the diseased growth.

The jugular veins were enormously dilated.

The descending cava is much increased in length, and passes through the tumour; on laying open three or four inches of it, it appears to have identified itself with the diseased mass, and in some parts small flattened tubercles with long peduncles, of a very soft texture, grow from its sides. The structure of the vein was so much altered in this part, as to present the appearance of the channel being continued through the tumour, and the venous tissue absorbed.

Right ventricle.-Cavity contracted, tricuspid valve shortened, in other respects normal.

Pulmonary artery.-Valves perfect. The left branch natural. The right passes through the tumour, and is much dilated, but retains its texture; portions of the tumour follow its course.

Left auricle* ${ }^{*}$ - The tumour in the interior of this 
cavity has made rapid progress. The substance of the auricle being absorbed to a considerable extent, the tumour presents the same uneven surface as in other parts. There are two distinct tubercles near the origin of the right pulmonary veins, the size of hazel-nuts with narrow peduncles; one of these is attached within the auricle, the peduncle of the other is attached to the inside of one of the right pulmonary veins.

The pulmonary veins of the left lung are free. One of those belonging to the right lung is dilated; its coats are very thin, and on making an incision we come to the substance of the tumour almost immediately. It is difficult to trace the other; it passes into the centre of the tumour and is lost.

Left ventricle small; mitral valve perfect. The aorta is diminished in calibre, the valves are entire.

The tumour occupied about one third of the cavity of the thorax, extending from the anterior to the posterior walls; it is formed of several irregular lobes of various sizes. It is attached to the trachea, bronchi, heart, great vessels, and right lung; it is moveable within the thorax. It appears to have no capsule, but a thin membrane covers it; except where it is closely applied to some of the before mentioned tissues.

The consistence is various; the hardest parts resemble soft cartilage, or portions of a scirrhous 
mamma : a considerable part is of a less firm consistence but of a solid character; a third part is almost pulpy and fluctuating, but retaining a cellular or fibrous appearance. On making a section of any part of the tumour a milk-white fluid escapes, resembling thick cream or mucilage of gum tragacanth, varying in quantity in proportion to the consistence of the part through which the incision is made. The firmest portions are contiguous to and within the lung; the softest between the trachea and bronchi, the great vessels, and attached to the auricles.

The appearance of the tumour both externally and internally very much resembles the oak-apple. The colour is almost entirely of a milk-white, in some places it is slightly tinged by the ramification of minute blood-vessels.

The number of blood-vessels is extremely small; in a great part none are visible carrying red blood. They are more numerous in the firmer parts attached to the lung.

One division of the tumour is very closely attached to the lung ; and this is the firmest portion of it, and has the most blood-vessels ramifying through it; in other parts the tumour is easily separated from the lung, and a membrane resembling the pleura appears to intervene.

On making sections in various directions into the 
firmer portion of the tumour, or that fixed to the lung, it presents a firm fibrous appearance; in the softer parts what have been termed cells, filled with the thick fluid, are very evident.

Abdomen.-The liver was very large and of the nutmeg appearance. No morbid alteration could be detected in the alimentary canal, spleen, pancreas, great vessels, absorbent glands, or urinary organs.

The model executed by Miller, and the drawings by Perry, now exhibited to the Society, give an exact representation of the disease.

The tumours described in the preceding cases, belong to the class of diseases ordinarily termed malignant.

The names of fungus hæmatodes, medullary sarcoma, cerebriform tumour, soft cancer, \&c., have been by many pathologists applied to them; whilst others have attempted to appropriate some of these names to distinct species of tumour; but the marks usually relied upon do not appear sufficient to warrant such a distinction.

In the case of M. K. several tissues and organs were affected with similar disease; in that of Imber; there was but one mass of disease, and the organs remote from the thorax did not participate in the same morbid change. 
In both cases the disease from its commencement appears to have increased rapidly; and although “ tumours of this description are, in the present state of our knowledge, essentially incurable, yet it is highly important" that pathologists should direct their attention to the investigation of the signs by which they may, with some degree of probability, be stpposed to exist during life. There are some striking symptoms adverted to in the foregoing cases, which led to the formation of a correct opinion of the character of the disease in these two instances, and which a more extensive series of cases might possibly confirm. If any advance can be made in a knowledge of the distinguishing marks of these tumours, the injurious and unavailing measures too frequently acopted, may be guarded against, and remedies which tend to palliate will be more diligently applied, and with a greater prospect of alleviating the extreme sufferings of our patients.

The immense tumour found in the thorax of Imber, does not consist in an alteration of any material structure, but is a new formation or adventitious growth.

The organ or tissue in which it commenced, may admit of various explanation. My impression is that it began in the lung, in the vicinity of the great vessels, and subsequently extended itself in other directions. In the firmest part, where the bloodvessels going into it are most numerous, the tumour 
adheres intimately to the lung. In this part, either in the substance of the lung or in the filamentous tissue connecting it with the adjoining parts, the tumour most probably originated, and the more soft and pulpy portions connected with the heart and great vessels may have been subsequently, and more rapidly formed.

We know nothing respecting the primary steps in the formation of tumours, but with regard to their mode of growth and propagation, a considerable advancement has been made.

On making sections of the firm parts of this tumour, very firm fibres enveloping a dense substance were observed, nearly resembling the sections of a cancerous mamma, and on dividing the pulpy parts, the usual appearances of cells was noticed, containing a thick cream-like fluid.

It appeared difficult to trace any approach to regularity in the formation of the several lobes of this tumour, but a minute inspection of it tends to favour the views proposed by Dr. Hodgkin, in his able and philosophical paper on adventitious structures.

In another case in which small tumours of cartilaginous hardness were found in several organs of the body, the appendix to the right auricle contained a 
mass of a similar kind, of unusual size. I shall relate it in addition to the foregoing cases.

\section{CASE III.}

Cartilaginous tumours in most of the tissues and organs, and involving the right auricle of the heart.

Sarah Fyfe, æt. 58, married; the mother of one child ; admitted into the Infirmary, June 25, 1831. She complains of pain over the abdomen, which is rather tumid and hard in the hypogastric region: constant and gnawing pain through the hips: urine scanty and high coloured, and great pain in emptying the bladder: no discharge from the vagina: surface of the body bloodless, extreme emaciation and debility. She has several small indurated moveable tumours situated in the skin covering various parts of the body.

About six months ago, whilst attending to domestic duties she was attacked with profuse discharge of blood by the vagina: this continued about a week, and was relieved by the remedies used. She had a fall about six weeks prior to this attack, but suffered little at the time from it. Since the above period, her health has rapidly declined, though she has had no return of the hæmorrhage. Her previous health had been delicate. 
27th. An examination per vaginam discovered round indurated masses about the os uteri.

The remedies which were used in some measure mitigated her sufferings; she, however, gradually became weaker, and died on July 4th.

July 5th. Dissection.-On dividing some of the small tumours on the surface of the body, they were of a cartilaginous hardness and uniform in texture.

Head.-A small quantity of fluid in the subaracnoid tissue and in the ventricles. Brain firm and remarkably pale : little or no blood appeared in the central or superficial vessels. A small indurated tumour in the falx of the dura mater. A tumour of a similar kind was situated in the right half of the pituitary gland, the size of a large pea. The carotid arteries in their canal were dilated and ossified. The posterior clynoid processes were thin and eroded, probably from the pressure of the arteries and the tumour of the pituitary gland.

Thorax.-Many tubercles were situated beneath the anterior part of the pleura costalis. Numerous similar tubercles were found on the pleuræ pulmonales, throughout the substance of the lungs. The lungs were edematous and studded with small miliary tubercles, and in the upper part of the right lung there were several cavities. A considerable quantity of fluid was found in the cavities of the pleuræ.

vol. XVIIr. 
Heart.-Slight hypertrophy : the left ventricle was dilated and softened.

Right auricle.-In the appendix to the right auricle there was a mass of adventitious deposit, the size of a small walnut.

Abdomen and Pelvis.-The liver and pancreas contained many tubercles of the character before mentioned: these with the other viscera of the abdomen, were, in other respects, natural.

Uterus. - The cervix was enlarged and indurated, and there was a small tumour projecting from the fundus into the cavity.

The filamentous tissue between the bladder and uterus was a good deal thickened and indurated, and of a similar appearance to that of the neck of the uterus.

Bladder. - The mucous membrane was generally vascular: about the anterior part of the neck large portions of the mucous membrane were detached, leaving a rough and vascular surface.

Since this paper was read to the Society I have met with another case of fungoid disease in the left lung. 
CASEIV.

Malignant tumour seated in the left lung. Extraordinary morbid change in the Pons Varolii.

Charles Jones, æt. 64, has been suffering hemiplegia of the left side about twelve months, and in consequence of the increase of cerebral disease was admitted into the St. Mary-le-bone Infirmary, July 23, 1833.

He has been subject to cough and other pulmonic symptoms for several years, but our attention was not at this time specially directed to the state of his chest, owing to the urgency of his other symptoms. Remedies afforded him but partial relief, and he died on the 6th of August.

Dissection 30 hours after death. Brain.-An extraordinary morbid alteration was observed in the, pons varolii : the particulars of the dissection of the brain, together with the symptoms referrible to it, I intend to relate on a future occasion.

Thorax.-In the upper portion of one of the lobes of the left lung was situated a tumour, the size of a small orange, it was imbedded in the centre of the lung, in the immediate vicinity of the bronchi and great vessel. Its surface was uneven with numerous rounded eminences. On making a section of the tumour, it proved to be of the fungoid or medullary character, and was composed of smaller lobules, 
300 DR. SIMS ON MATIGMANT TUMOURS, ETC.

in some parts presenting a high degree of vascularity. The lungs were edematous and loaded with blood. Traces of chronic pneumonia, in various stages, were observed, with copious infiltration of greenish fluid into the pulmonary tissue, in some places resembling gangrenous patches. There was extensive solid grey hepatization near the root of the left lung, where the tumour was situated.

\section{Heart flabby, cavities and valves natural.}

In the abdominal viscera no morbid change was noticed, except a large scrotal hernia, in which the intestine was confined by strong lengthened bands of membrane.

END OF PART I. 